\title{
A Phase I-II Basket Trial Design to Optimize Dose-Schedule Regimes Based on Delayed Outcomes $^{\S}$
}

\author{
Ruitao Lin*, Peter F. Thall ${ }^{\dagger}$, and Ying Yuan ${ }^{\ddagger}$
}

\begin{abstract}
This paper proposes a Bayesian adaptive basket trial design to optimize the dose-schedule regimes of an experimental agent within disease subtypes, called "baskets", for phase I-II clinical trials based on late-onset efficacy and toxicity. To characterize the association among the baskets and regimes, a Bayesian hierarchical model is assumed that includes a heterogeneity parameter, adaptively updated during the trial, that quantifies information shared across baskets. To account for late-onset outcomes when doing sequential decision making, unobserved outcomes are treated as missing values and imputed by exploiting early biomarker and low-grade toxicity information. Elicited joint utilities of efficacy and toxicity are used for decision making. Patients are randomized adaptively to regimes while accounting for baskets, with randomization probabilities proportional to the posterior probability of achieving maximum utility. Simulations are presented to assess the design's robustness and ability to identify optimal dose-schedule regimes within disease subtypes, and to compare it to a simplified design that treats the subtypes independently.
\end{abstract}

MSC2020 subject classifications: Primary 60K35, 60K35; secondary 60K35.

Keywords: adaptive randomization, Bayesian design, basket trial, missing data, optimal treatment regime, phase I-II clinical trial.

\section{Introduction}

Early-phase oncology clinical trials were traditionally designed to evaluate new treatments under the assumption that patients are homogeneous. Advances in cancer biology and genomic medicine have shifted the focus of cancer research and therapy from conventional chemotherapy to agents that target specific genetic or molecular abnormalities (Simon and Roychowdhury, 2013). Because different cancer histologies may share a common target, this motivates the evaluation of different cancers within the

${ }^{*}$ Department of Biostatistics, The University of Texas MD Anderson Cancer Center, Houston, Texas 77030, U.S.A., rlin@mdanderson.org

${ }^{\dagger}$ Department of Biostatistics, The University of Texas MD Anderson Cancer Center, Houston, Texas 77030, U.S.A., rex@mdanderson.org

${ }_{\ddagger}^{\ddagger}$ Department of Biostatistics, The University of Texas MD Anderson Cancer Center, Houston, Texas 77030, U.S.A., yyuan@mdanderson.org

$\S$ The authors thank the handling Editor, the Associate Editor, the two referees, and the Editor for their many constructive and insightful comments that have led to significant improvements in the article. RL was funded by NIH/NCI grants P30 CA016672 and P50 CA221703, PFT was funded by NIH/NCI grants P30 CA016672 and P01 CA148600, and YY was funded by NIH/NCI grants P50 CA098258, P50 CA217685, and P50 CA221707. 
same clinical trial. To accommodate this approach in the early-phase evaluation of a new targeted agent, basket trials have emerged as a way to account for different disease subtypes (Redig and Jänne, 2015; Ornes, 2016). Basket trials provide an approach that is intermediate between conducting separate trials within cancer subtypes and ignoring subtypes entirely. Compared to traditional early-phase trial designs, a basket trial has the advantages of borrowing strength between disease subtypes, which may improve the efficiency of the trial in terms of sample size and trial duration (Simon et al., 2016), and also may allow for the inclusion of patients with rare cancers.

Several adaptive basket trial designs have been proposed. Thall et al. (2003) and Berry et al. (2013) used hierarchical models to borrow information across different cancer subtypes. Simon et al. (2016) proposed a Bayesian model that includes a parameter to quantify heterogeneity of treatment effects across disease subtypes. Cunanan et al. (2017) proposed an efficient two-stage basket trial design. Trippa and Alexander (2017) proposed using adaptive randomization (AR) in a Bayesian basket trial design. Chu and Yuan (2018a) proposed a calibrated Bayesian hierarchical model to improve performance. Chu and Yuan (2018b) proposed a Bayesian latent-class design to account for subtype heterogeneity by adaptively grouping the disease subtypes into clusters based on their treatment responses, and then borrowing information within the clusters using a Bayesian hierarchical model.

Our research is motivated by a planned phase I-II trial to optimize the (dose, schedule) regime of PGF melphalan as a single agent preparative regimen for autologous stem cell transplantation (autosct) in patients with multiple myeloma (MM). This disease is heterogeneous, with several different classification systems, studied by Zhang et al. (2006). Most commonly, MM is dichotomized as hyperdiploid or not, in terms of pathogenesis pathways defined by genetic and cytogenetic abnormalities. A review is given by Fonseca et al. (2009). The primary objective of our motivating trial is to determine the optimal (dose, schedule) treatment regime for each MM subtype by using efficacy and toxicity as co-primary endpoints (Thall et al., 2013; Yuan et al., 2016). The trial will study three PGF melphalan doses, 200,225 and $250 \mathrm{mg} / \mathrm{m}^{2}$, and three infusion schedules, 30 minutes, 12 hours, and 24 hours, yielding nine treatment regimes. Toxicity is defined as the binary indicator of grade 3 mucositis lasting $>3$ days or any grade 4 (severe) or 5 (fatal) non-hematologic or non-infectious toxicity, with onset within 30 days from the start of treatment infusion. In particular, a patient cannot be assessed as having "no toxicity" until he/she has been followed for 30 days. Efficacy is defined as the binary indicator of complete remission, evaluated at day 90 . Thus, toxicity may be observed soon enough to feasibly apply a sequential toxicity-based decision rule, but the efficacy outcome is evaluated much later. Even if the accrual rate is moderately fast, a substantial number of treated patients will not have had their efficacy outcomes assessed, and some patients will not have had their toxicity outcomes assessed, at the time that treatment regimes must be chosen for newly enrolled patients. This is a major logistical difficulty when making outcome-adaptive decisions for new patients, including choosing (dose, schedule) or determining whether a treatment regime is unsafe. Furthermore, these adaptive decisions must be made for each MM subgroup.

In this paper, we propose an efficient basket design for adaptively optimizing doseschedule regimes, and conducting safety monitoring, within disease subtypes in phase 
I-II trials with late-onset (toxicity, efficacy) outcomes. This problem has not been considered by existing methods for basket trials. In the MM trial, the design allows patients with different subtypes to be given different dose-schedule regimes, an example of "precision medicine." In phase I-II trials with heterogeneous patients, a major concern is whether the dose-efficacy or dose-toxicity curves differ between disease subtypes. This is more complex than basing decisions on one-dimensional treatment effects, which are the basis for existing basket trial designs. Dealing with multi-dimensional outcomes is challenging in early phase trial designs. See, for example, Lee et al. (2019).

The MM trial is complicated by the following four issues: (1) Adaptive treatment decisions must account for the relationships between efficacy, toxicity, dose, schedule, and disease subtype. (2) For each disease subtype, the $\operatorname{Pr}$ (efficacy | dose, schedule, subtype) function may take a variety of possible forms that may or may not be monotonic in dose. Nearly all existing subtype-specific phase I dose-finding designs assume monotonic increasing dose-toxicity curves. See, for example, Morita et al. (2017), Chapple and Thall (2018). (3) In the MM trial, because efficacy is scored at day 90 from the start of therapy, no efficacy data for patients who have been followed for less than 90 days are available, but it is not feasible to suspend accrual until all previously treated patients' outcomes are fully observed, to apply outcome-adaptive rules. Thus, we may not use existing adaptive methods that consider only time-to-event outcomes and use follow-up time without efficacy as partial information, as in the designs of Cheung and Chappell (2000) and Yuan and Yin (2011). (4) Borrowing information across subtypes is not straightforward, because it is unknown a priori whether (dose, schedule) effects are homogeneous or heterogeneous between subtypes.

To construct a practical design that addresses all of these issues, we assume a flexible three-level Bayesian hierarchical model to characterize the associations among dose, schedule, disease subtype, and the (toxicity, efficacy) outcome. The hierarchical model facilitates borrowing information adaptively across subtypes. To improve the probability of identifying optimal (dose, schedule) regimes within subtypes, the design repeatedly determines whether or not treatment effects are homogeneous across subtypes. We treat temporarily unobserved ("late-onset") outcomes as missing values, and impute them by exploiting auxiliary information that is observed sooner, including low-grade toxicity and bioactivity data. This substantially improves efficiency when such auxiliary outcomes are informative. Elicited utilities of (toxicity, efficacy) outcomes are used as a basis for sequentially adaptive subtype-specific (dose, schedule) optimization. To avoid getting stuck at a suboptimal regime, our proposed two-stage design adaptively randomizes each newly enrolled patient to a treatment regime according to that patient's disease subtype.

A simpler design is proposed by Lin et al. (2020a) to optimize dose-schedule regimes in a similar setting, but it assumes that the probability of treatment efficacy is strictly ordered for different subtypes, and it does not borrow any additional information to deal with delayed outcomes. In contrast, the design proposed in here is more general in that (1) it does not make any ordering assumption about the probability of efficacy, (2) it can adaptively identify response homogeneity or heterogeneity across subtypes based on the observed data, and (3) it uses more of the available data, including bioactivity and 
low-grade toxicity data, in making treatment decisions when some previously treated patients have efficacy outcome data pending.

To make things concrete, we present the design in the context of the motivating trial. The design potentially can be applied quite widely, however. In many early phase oncology trials, the efficacy outcome is defined to be evaluated a substantial amount of time after the start of therapy. Moreover, it is routine practice to define toxicity as an ordinal categorical variable, in terms of severity grade, and to record biological or conventional prognostic variables related to efficacy prior to enrollment.

The remainder of this paper is organized as follows. In Section 2, we present the hierarchical model for the subtype-dose-schedule-response relationship and the Bayesian data augmentation model for unobserved outcomes. In Section 3, we describe the trial design, including the utility function, rules for trial conduct, and prior elicitation methods. In Section 4, we apply the proposed design to the motivating trial and conduct simulation studies to examine the design's performance. We close with a brief discussion in Section 5 and provide other technical details and the results of additional simulations in the Supplementary Material (Lin et al., 2020b). The R code to implement and simulate the proposed design is available from the first author upon request.

\section{Probability Model}

\subsection{Inference Model}

We consider a phase I-II trial to evaluate all combinations of $D$ doses and $S$ treatment schedules, for a total of $D S$ treatment regimes, where each patient has one of $B$ different tumor subtypes, known as "baskets." Let $n$ denote the number of patients accrued by an interim decision-making point in the trial, and index patients by $i=1, \ldots, n$. For the $i^{t h}$ patient, denote toxicity by $X_{i}$, efficacy by $Y_{i}$, cancer subtype by $b_{i} \in\{1, \ldots, B\}$, and the assigned dose-schedule treatment regime by $r_{i}=\left(d_{i}, s_{i}\right)$, for $d_{i} \in\{1, \ldots, D\}$, and $s_{i} \in\{1, \ldots, S\}$. We assume that $X_{i}$ and $Y_{i}$ both are binary, with $X_{i}=1$ indicating dose-limiting toxicity (DLT) and $Y_{i}=1$ indicating response. However, toxicity may occur and be observed at any time during a predefined assessment window $\left[0, T_{X}\right]$ and, similarly, efficacy is either observed at some time during a window $\left[0, T_{Y}\right]$ or observed at $T_{Y}$. This is similar to the outcome structures considered in a phase I-II setting by Jin et al. (2014). Extension of this structure to accommodate bivariate ordinal outcomes, as in Thall et al. (2017), is conceptually straightforward but technically much more complex.

To characterize the joint distribution of the observed outcomes $\left(X_{i}, Y_{i}\right)$ as a function of regime $r_{i}$ and disease subtype $b_{i}$, we propose a three-level Bayesian hierarchical model. This is more elaborate than a more conventional two-level hierarchical model, and is motivated by the desire to account for (1) effects of latent variables used to define the observed outcomes, done in the Level 1 model, and also (2) joint effects of patient subgroups and treatment regimes, done in the Level 2 model. This may be regarded as an extra level in the hierarchy that accounts for between-patient variability while borrowing information between subtypes by assuming that the mean (subtype, schedule) 
effects are iid across disease subtypes. Level 3 then provides priors for mean and variance parameters appearing in Level 2 of the hierarchical model.

Formally, following Albert and Chib (1993), Chen and Dey (1998), and Chib and Greenberg (1998), as a device to facilitate joint modeling and computation, we define each observed $\left(X_{i}, Y_{i}\right)$ pair in terms of real-valued bivariate normal latent variables, $\left(\xi_{i}, \eta_{i}\right)$, as $X_{i}=\mathrm{I}\left(\xi_{i}>0\right)$ and $Y_{i}=\mathrm{I}\left(\eta_{i}>0\right)$, where $\mathrm{I}(A)$ denotes the indicator function for the event $A$. Thus, the joint distribution of $\left(X_{i}, Y_{i}\right)$ is induced by that of $\left(\xi_{i}, \eta_{i}\right)$. The three-level hierarchical model that we use to specify the distribution of the latent variables $\left(\xi_{i}, \eta_{i}\right)$, for disease subtype $b_{i}$, and treatment regime $r_{i}=\left(d_{i}, s_{i}\right)$, is as follows:

$$
\begin{array}{ll}
\text { Level 1: } & \xi_{i} \mid b_{i}, r_{i}, u_{i}, \tilde{\xi}_{b_{i}, r_{i}}, \sigma_{\xi}^{2} \stackrel{\text { ind }}{\sim} \mathrm{N}\left(\tilde{\xi}_{b_{i}, r_{i}}+u_{i}, \sigma_{\xi}^{2}\right), \quad i=1, \ldots, n \\
& \eta_{i} \mid b_{i}, r_{i}, v_{i}, \tilde{\eta}_{b_{i}, r_{i}}, \sigma_{\eta}^{2} \stackrel{\text { ind }}{\sim} \mathrm{N}\left(\tilde{\eta}_{b_{i}, r_{i}}+v_{i}, \sigma_{\eta}^{2}\right), \quad i=1, \ldots, n \\
\text { Level 2: } & u_{i}, v_{i} \mid \Sigma_{u, v} \stackrel{\text { i.i.i.d. }}{\sim} \mathrm{BN}\left(\mathbf{0}_{2}, \Sigma_{u, v}\right), \quad i=1, \ldots, n \\
& \tilde{\xi}_{b, r} \mid \ddot{\xi}_{r}, \tau_{\xi} \stackrel{\text { i.i.d. }}{\sim} \mathrm{N}\left(\ddot{\xi}_{r}, \tau_{\xi}^{2}\right), \quad b=1, \ldots, B \\
& \tilde{\eta}_{b, r} \mid \ddot{\eta}_{r}, \tau_{\eta} \stackrel{\text { i.i.d. }}{\sim} \mathrm{N}\left(\ddot{\eta}_{r}, \tau_{\eta}^{2}\right), \quad b=1, \ldots, B \\
\text { Level 3: } & \ddot{\xi}_{d, s} \mid \ddot{\boldsymbol{\xi}}_{-d, s}, \xi_{0}, \nu_{\xi}^{2} \stackrel{\text { i.i.d. }}{\sim} \mathrm{N}\left(\xi_{0}, \nu_{\xi}^{2}\right) \mathrm{I}\left(\ddot{\xi}_{d-1, s}<\ddot{\xi}_{d, s}<\ddot{\xi}_{d+1, s}\right), \quad d=1, \ldots, D \\
& \ddot{\eta}_{d, s} \mid \eta_{0}, \nu_{\eta}^{2} \stackrel{\text { i.i.d. }}{\sim} \mathrm{N}\left(\eta_{0}, \nu_{\eta}^{2}\right), \quad d=1, \ldots, D \\
& \tau_{\xi}, \tau_{\eta} \mid \psi_{0}, \gamma_{0} \stackrel{\text { ind }}{\sim} \text { half-Cauchy }\left(\psi_{0}, \gamma_{0}\right) .
\end{array}
$$

Above, $\mathrm{BN}\left(\mathbf{0}_{2}, \Sigma_{u, v}\right)$ denotes a bivariate normal distribution with mean vector $\mathbf{0}_{2}=$ $(0,0)$ and covariance matrix $\Sigma_{u, v}, \ddot{\boldsymbol{\xi}}_{-d, s}$ denotes the subvector of $\ddot{\boldsymbol{\xi}}_{s}=\left(\ddot{\xi}_{1, s}, \ldots, \ddot{\xi}_{D, s}\right)$ with $\ddot{\xi}_{d, s}$ deleted for $d=1, \ldots, D$, and $\mathrm{N}(\cdot) \mathrm{I}(A)$ denotes the truncated normal distribution having support given by the set $A$ in the indicator function. We denote the vector of all fixed hyperparameters that must be prespecified by $\boldsymbol{\theta}_{0}=\left(\sigma_{\xi}^{2}, \sigma_{\eta}^{2}, \Sigma_{u, v}, \xi_{0}, \eta_{0}, \nu_{\xi}^{2}, \nu_{\eta}^{2}\right.$, $\left.\psi_{0}, \gamma_{0}\right)$.

In Level 1 of the model, the patient-specific random effects $\left(u_{i}, v_{i}\right)$ induce association between $\xi_{i}$ and $\eta_{i}$, which in turn induces the association between $X_{i}$ and $Y_{i}$. Numerical values of the hyperparameters $\left(\sigma_{\xi}^{2}, \sigma_{\eta}^{2}\right)$ must be specified to ensure that the model is identifiable. The Level 2 priors on $\tilde{\xi}_{b, r}$ and $\tilde{\eta}_{b, r}$ facilitate information borrowing across cancer subtypes. Specifically, for each (dose, schedule) regime $r$, we assume that $\left\{\tilde{\xi}_{b, r}, b=1, \cdots, C\right\}$ (or $\left\{\tilde{\eta}_{b, r}, b=1, \cdots, C\right\}$ ) are generated from a common normal distribution, where $\left(\ddot{\xi}_{r}, \ddot{\eta}_{r}\right)$ are the mean (toxicity, efficacy) effects of regime $r=(d, s)$, and $\left(\tau_{\xi}^{2}, \tau_{\eta}^{2}\right)$ characterize the degree of heterogeneity of toxicity and efficacy, respectively, between subtypes. As $\tau_{\xi}^{2}, \tau_{\eta}^{2} \rightarrow 0$, model (2.1) shrinks to the homogeneous case for which the regime effects in different subtypes are the same. In the motivating MM trial, based on clinical experience the toxicity distribution is assumed to be homogeneous between subgroups, but efficacy may be heterogeneous between subgroups. We thus simplify the model by setting $\tau_{\xi}^{2}=0$, while $\tau_{\eta}^{2} \neq 0$. In general, however, the model can account for heterogeneous toxicity by specifying $\tau_{\xi}^{2} \neq 0$. 
Since $\tau_{\eta}^{2}$ controls the degree to which efficacy information is borrowed across different tumor subtypes, rather than fixing $\tau_{\eta}^{2}$, we estimate its value adaptively based on the observed data. The choice of the prior on $\tau_{\eta}^{2}$ is critical to ensure good performance of the proposed design. To leverage information sharing, we follow the suggestion of Gelman (2006) by assuming a half-Cauchy $\left(\psi_{0}, \gamma_{0}\right)$ prior on $\tau_{\eta}$ in Level 3 of the model, where $\psi_{0}$ is the location parameter and $\gamma_{0}$ is the scale parameter. When $\psi_{0}=0$ and $\gamma_{0}$ is large, this prior is weakly informative. To account for the common assumption that the risk of toxicity increases monotonically with dose for each schedule, in the Level 3 prior we impose an order constraint on $\left\{\ddot{\xi}_{d, s}, d=1, \ldots, D\right\}$ for each $s$ at each Markov chain Monte Carlo sampling step. This ensures that the latent variable for toxicity increases stochastically in $d$. In contrast, we do not impose any monotonicity restriction on efficacy, so that the dose-efficacy curve is very flexible and can take a wide variety of shapes. In the MM trial, there is no ordering based on infusion schedule. If the infusion times would affect the risk of toxicity in other settings, however, such prior ordering information also can be similarly incorporated in our design.

Under the hierarchical model (2.1), given $\left(\tilde{\xi}_{b_{i}, r_{i}}, \tilde{\eta}_{b_{i}, r_{i}}\right)$, the joint distribution of $\left(\xi_{i}, \eta_{i}\right)$ can be obtained by integrating out $\left(u_{i}, v_{i}\right)$, which yields

$$
\xi_{i}, \eta_{i} \mid \boldsymbol{\mu}_{b_{i}, r_{i}}, \Sigma_{\xi, \eta} \stackrel{\text { ind }}{\sim} \mathrm{BN}\left(\boldsymbol{\mu}_{b_{i}, r_{i}}, \Sigma_{\xi, \eta}\right), \quad i=1, \ldots, n .
$$

The mean vector $\boldsymbol{\mu}_{b_{i}, r_{i}}$ depends on both the $i^{\text {th }}$ patient's cancer subtype $b_{i}$ and the treatment regime $r_{i}=\left(d_{i}, s_{i}\right)$, with $\boldsymbol{\mu}_{b_{i}, r_{i}}=\left(\tilde{\xi}_{b_{i}, r_{i}}, \tilde{\eta}_{b_{i}, r_{i}}\right)$, and

$$
\Sigma_{\xi, \eta}=\Sigma_{u, v}+\left[\begin{array}{cc}
\sigma_{\xi}^{2} & 0 \\
0 & \sigma_{\eta}^{2}
\end{array}\right]
$$

Suppose, temporarily, that the data of the first $n$ patients are completely observed, and let $D_{n}^{\text {com }}=\left\{\left(x_{i}, y_{i}\right), i=1, \ldots, n\right\}$ denote the complete dataset and $\boldsymbol{\theta}=\left\{\boldsymbol{\mu}_{b, r}, b=\right.$ $1, \ldots, B, d=1, \ldots, D, s=1, \ldots, S\} \cup \Sigma_{\xi, \eta}$. The complete data likelihood, based on $D_{n}^{\text {com }}$, is given by

$$
L\left(\boldsymbol{\theta} \mid D_{n}^{\mathrm{com}}\right)=\prod_{i=1}^{n} \int_{\ell_{x_{i}}}^{\ell_{x_{i}+1}} \int_{\ell_{y_{i}}}^{\ell_{y_{i}+1}} f\left(\xi_{i}, \eta_{i} \mid \boldsymbol{\mu}_{b_{i}, r_{i}}, \Sigma_{\xi, \eta}\right) \mathrm{d} \eta_{i} \mathrm{~d} \xi_{i},
$$

where $x_{i}, y_{i}=0,1, f\left(\xi_{i}, \eta_{i} \mid \boldsymbol{\mu}_{b_{i}, r_{i}}, \Sigma_{\xi, \eta}\right)$ is the distribution induced by (2.2), and the cutoff vector $\left(\ell_{0}, \ell_{1}, \ell_{2}\right)=(-\infty, 0, \infty)$. Let $\pi\left(\boldsymbol{\theta} \mid \boldsymbol{\theta}_{0}\right)$ be the joint prior distribution on $\boldsymbol{\theta}$ based on the hierarchical model (2.1). The joint posterior of $\boldsymbol{\theta}$ is given by $\pi(\boldsymbol{\theta} \mid$ $\left.\boldsymbol{\theta}_{0}, D_{n}^{\text {com }}\right) \propto \pi\left(\boldsymbol{\theta} \mid \boldsymbol{\theta}_{0}\right) L\left(\boldsymbol{\theta} \mid D_{n}^{\text {com }}\right)$.

\subsection{Imputation Model}

When the accrual of new patients is fast relative to the duration of the toxicity and efficacy assessment periods, $T_{X}$ and $T_{Y}$, there will be some patients for whom $X_{i}$ and $Y_{i}$ are not known at the interim time when adaptive decisions must be made for newly enrolled patients. In the MM trial, the toxicity assessment window is $\left[0, T_{X}\right]=[0,30]$ 
days, while efficacy is defined as complete remission at the $T_{Y}=90$ day evaluation. If, for example, the accrual rate is 6 patients per month, then an average of 18 new patients will be accrued while waiting to evaluate $Y_{i}$ for the last enrolled patient. In other words, at the time of decision making, both the toxicity and efficacy data of previously treated patients are subject to temporary missingness, which is nonignorable (Liu et al., 2013). Once the patients who have pending $Y_{i}$ values reach their 90-day assessment times, their (toxicity, efficacy) outcomes have been observed completely. If the accrual were suspended repeatedly until all previously treated patients were fully assessed, this would lead to an impractically lengthy trial, and would greatly delay the treatment of new patients, which cannot be done in an actual trial. To address this realistically, we exploit additional interim auxiliary information related to the unobserved $\left(X_{i}, Y_{i}\right)$, as follows.

Let $t_{i} \leq \max \left\{T_{X}, T_{Y}\right\}$ denote the follow-up time of patient $i$, within the observation windows, at some interim decision-making time. Let $U_{i}$ denote the time to toxicity, with $\boldsymbol{\delta}=\left(\delta_{X, i}, \delta_{Y, i}\right)$ the response indicator vector of whether each outcome of the $i^{\text {th }}$ subject has been observed by $t_{i}$. The binary toxicity outcome $X_{i}$ is observed if $U_{i} \leq t_{i} \leq T_{X}$, so $X_{i}=1$, in which case $\delta_{X, i}=1$, or the patient has finished the assessment without experiencing toxicity, i.e., $t_{i} \geq T_{X}$ and $U_{i}>T_{X}$, so $X_{i}=0$. In contrast, the efficacy outcome is observed if and only if the patient has reached their efficacy assessment time, that is, $\delta_{Y, i}=\mathrm{I}\left(t_{i} \geq T_{Y}\right)$. For example, if $t_{i}<U_{i}$ then $X_{i}$ is missing and $\delta_{X, i}=0$ at $t_{i}$. Therefore, the observed data for the first $n$ patients are $D_{n}^{\mathrm{obs}}=\left\{\left(\delta_{X, i}, \delta_{Y, i}, t_{i}, \tilde{X}_{i}, \tilde{Y}_{i}\right), i=1, \ldots, n\right\}$, where $\tilde{X}_{i}=\delta_{X, i} X_{i}$ and $\tilde{Y}_{i}=\delta_{Y, i} Y_{i}$.

To exploit information on low grade toxicity that may be available before the binary toxicity outcome $X_{i}$ is observed, we define a binary indicator, $L_{i}$, of whether the $i^{\text {th }}$ patient has experienced low-grade toxicity $\left(L_{i}=1\right)$ or not $\left(L_{i}=0\right)$. We assume that a patient with low-grade toxicity is more likely to experience DLT, formally $\operatorname{Pr}\left(X_{i}=1\right.$ | $\left.L_{i}=1\right)>\operatorname{Pr}\left(X_{i}=1 \mid L_{i}=0\right)$, so $L_{i}$ may be used to help predict as yet unobserved $X_{i}$. In our example, $L_{i}$ is available much sooner than $T_{X}$. We assume that, if a patient has finished the toxicity assessment without experiencing any DLT, then his/her timeto-toxicity outcome is censored at $T_{X}$. The observed event indicator is $\tilde{X}_{i}$, with $\tilde{X}_{i}=1$ if that patient $i$ had toxicity, and $\tilde{X}_{i}=0$ if the time to toxicity for patient $i$ is censored by the follow-up time $t_{i}$ or maximum toxicity assessment time $T_{X}$. Formally, $\tilde{X}_{i}=$ $\mathrm{I}\left\{U_{i} \leq \min \left(t_{i}, T_{X}\right)\right\}$. We assume the following proportional hazards (PH) model for the distribution of the time to toxicity.

$$
\begin{aligned}
\text { PH model : } & \lambda\left(U_{i} \mid L_{i}\right)=\lambda_{0}\left(U_{i}\right) \exp \left(\beta L_{i}\right), \quad i=1, \ldots, n, \\
\text { Baseline model : } & U_{i} \mid L_{i}=0, p, q \stackrel{\text { i.i.d }}{\sim} \operatorname{Weibull}(p, q), \quad i=1, \ldots, n, \\
\text { Priors : } & \beta \mid \sigma_{\beta} \sim \mathrm{N}(0, \sigma \beta) \mathrm{I}(0, \infty), \\
& p\left|\alpha_{p}, \beta_{p} \sim \operatorname{Gamma}\left(\alpha_{p}, \beta_{p}\right), q\right| \alpha_{q}, \beta_{q} \sim \operatorname{Gamma}\left(\alpha_{q}, \beta_{q}\right),
\end{aligned}
$$

where $\lambda\left(U_{i} \mid L_{i}\right)$ is the conditional hazard function of the time to toxicity given the lowgrade toxicity indicator $L_{i}, \lambda_{0}\left(U_{i}\right)$ is the baseline hazard, and $\boldsymbol{\theta}_{1}=\left(\sigma_{\beta}, \alpha_{p}, \beta_{p}, \alpha_{q}, \beta_{q}\right)$ are hyperparameters. We assume that the baseline survival follows a Weibull distribution, which is flexible enough to characterize the time-to-toxicity data in our MM 
setting. Let $f\left(U_{i} \mid L_{i}, p, q, \beta\right)$ be the density of $U_{i}$ and denote the joint posterior distribution

$$
\begin{aligned}
\pi\left(p, q, \beta \mid D_{n}^{\mathrm{obs}}, \boldsymbol{\theta}_{1}\right) & \propto \pi\left(p, q, \beta \mid \boldsymbol{\theta}_{1}\right) L_{T}\left(D_{n}^{\mathrm{obs}} \mid p, q, \beta\right) \\
& =\pi\left(p, q, \beta \mid \boldsymbol{\theta}_{1}\right) \prod_{i=1}^{n} f\left(t_{i} \mid L_{i}, p, q, \beta\right)^{\tilde{X}_{i}} S\left(\min \left(t_{i}, T_{X}\right) \mid L_{i}, p, q, \beta\right)^{\left(1-\tilde{X}_{i}\right)},
\end{aligned}
$$

where $S\left(\cdot \mid L_{i}, p, q, \beta\right)$ is the survival function. After obtaining the posterior distribution $\pi\left(p, q, \beta \mid D_{n}^{\text {obs }}, \boldsymbol{\theta}_{1}\right)$, the missing toxicity outcome with $\delta_{X, i}=0$ can be imputed by a Bernoulli random variable with the probability of DLT given by

$$
\begin{aligned}
\operatorname{Pr}\left(X_{i}=1 \mid \delta_{X, i}=0, D_{n}^{\text {obs }}, p, q, \beta\right) & =\operatorname{Pr}\left(U_{i} \leq T_{X} \mid U_{i} \geq t_{i}, D_{n}^{\text {obs }}, p, q, \beta\right) \\
& =\frac{S\left(t_{i} \mid l_{i}, p, q, \beta\right)-S\left(T_{X} \mid l_{i}, p, q, \beta\right)}{S\left(t_{i} \mid l_{i}, p, q, \beta\right)} .
\end{aligned}
$$

In the MM trial, 90-day response is defined as complete response (CR) or partial response (PR). In therapy of $\mathrm{MM}, \mathrm{CR}$ is defined as is negative immunofixation on analysis of blood serum and urine, disappearance of any soft tissue plasmacytomas, and $<5 \%$ plasma cells in the bone marrow, and PR is defined as $\geq 50 \%$ of blood serum M-protein and $\geq 90 \%$ reduction in urinary M-protein level within 24 hours. Additional details are given by Durie et al. (2006). Thus, early measurement of the M-protein in the serum and urine are associated with the 90-day response indicator $Y_{i}$.

Let $Z_{i k}$ denote the biomarker measurement for the $i^{\text {th }}$ patient at time $t_{i k}^{\prime}, k=$ $1, \ldots, K_{i}$, where $K_{i}$ is the total number of biomarker measurements of the $i^{t h}$ patient by the decision-making time $t_{i}$. We model the longitudinal biomarker measurements $Z_{i k}$ and link them to the efficacy outcome $Y_{i}$ using the following hierarchical model.

$$
\begin{aligned}
\text { Bioactivity Model: } & Z_{i k} \mid t_{i k}^{\prime}, w_{0}, w_{b_{i}, r_{i}}, \sigma_{z}^{2} \stackrel{\text { ind }}{\sim} \mathrm{N}\left(w_{0}+w_{b_{i}, r_{i}} t_{i k}^{\prime}, \sigma_{z}^{2}\right), \\
\text { Link Model : } & \eta_{i} \mid w_{b_{i}, r_{i}}, \boldsymbol{\alpha}, \sigma_{\eta}^{2} \stackrel{\text { ind }}{\sim} \mathrm{N}\left(\alpha_{0}+\alpha_{1} w_{b_{i}, r_{i}}+\alpha_{2} w_{b_{i}, r_{i}}^{2}, \sigma_{\eta}^{2}\right), \\
\text { Level 1 Priors: } & w_{b, r} \mid \bar{w}_{r}, \tau_{w}^{2} \stackrel{\text { i.i.i.d. }}{\sim} \mathrm{N}\left(\bar{w}_{r}, \tau_{w}^{2}\right), \quad b=1, \ldots, B \\
& w_{0} \mid \tau_{w_{0}}^{2} \sim \mathrm{N}\left(0, \tau_{w_{0}}^{2}\right), \\
& \alpha_{1}, \alpha_{2} \mid \tau_{\alpha}^{2} \stackrel{\text { i.i.i.d. }}{\sim} \mathrm{N}\left(0, \tau_{\alpha}^{2}\right), \\
& \sigma_{z}^{2} \sim \operatorname{IGamma}\left(\alpha_{z}, \beta_{z}\right), \\
\text { Level 2 Priors: } \quad & \bar{w}_{r} \mid \bar{w}_{0}, \nu_{w}^{2} \stackrel{\text { i.i.d. }}{\sim} \mathrm{N}\left(\bar{w}_{0}, \nu_{w}^{2}\right), \\
& \tau_{w}^{2} \sim \text { half-Cauchy }\left(\psi_{0}, \gamma_{0}\right),
\end{aligned}
$$

where $\boldsymbol{\alpha}=\left(\alpha_{0}, \alpha_{1}, \alpha_{2}\right)$ and $\boldsymbol{\theta}_{2}=\left(\sigma_{\eta}^{2}, \alpha_{0}, \tau_{w_{0}}^{2}, \tau_{\alpha}^{2}, \alpha_{z}, \beta_{z}, \bar{w}_{0}, \nu_{w}^{2}, \psi_{0}, \gamma_{0}\right)$ are hyperparameters. In particular, $\alpha_{0}$ must be fixed to make the model identifiable. In the bioactivity model, $w_{0}$ is the intercept, which can be viewed as the baseline biomarker value, and the second term $w_{b_{i}, r_{i}} t_{i k}^{\prime}$ captures the trajectory of the biomarker. The relationship between the latent variable $\eta_{i}$ and the random effect $w_{b_{i}, r_{i}}$ induces association between the biomarker measurements and the efficacy outcome. The random effect $w_{b, r}$ 
depends on the patient's disease subtype and treatment regime. We also assume that $\left\{w_{b, r}, b=1, \ldots, B\right\}$ are sampled from a common normal distribution with variance parameter $\nu_{w}^{2}$, which determines the amount of information shared across subtypes. In other words, $\nu_{w}^{2}$ is another heterogeneity parameter derived from the bioactivity data.

Denote $\mathbf{w}=\left\{w_{b, r}\right\}$ for $b=1, \ldots, B$ and all $D S$ pairs $r=(d, s)$. We denote the posterior distribution of $(\boldsymbol{\alpha}, \mathbf{w})$ under the Bayesian hierarchical model (2.4) by $\pi(\boldsymbol{\alpha}, \mathbf{w}$ $\left.\boldsymbol{\theta}_{2}, \tilde{D}_{n}^{\text {obs }}\right)$, where $\tilde{D}_{n}^{\text {obs }}=D_{n}^{\text {obs }} \cup\left\{\mathbf{z}_{i}, i=1, \ldots, n\right\}$ with $\mathbf{z}_{i}=\left\{z_{i k}, k=1, \ldots, K_{i}\right\}$. A missing efficacy outcome, with $\delta_{Y, i}=0$, can be imputed as a Bernoulli random variable with success probability

$$
\operatorname{Pr}\left(Y_{i}=1 \mid \delta_{Y, i}=0, \tilde{D}_{n}^{\text {obs }}, \boldsymbol{\alpha}, \mathbf{w}, \sigma_{\eta}^{2}\right)=\int_{\ell_{1}}^{\ell_{2}} f\left(\eta_{i} \mid \tilde{D}_{n}^{\text {obs }}, \boldsymbol{\alpha}, \mathbf{w}, \sigma_{\eta}^{2}\right) \mathrm{d} \eta_{i},
$$

where the conditional distribution $f\left(\eta_{i} \mid \tilde{D}_{n}^{\text {obs }}, \boldsymbol{\alpha}, \mathbf{w}, \sigma_{\eta}^{2}\right)$ is derived from (2.4).

To deal with temporarily missing toxicity/efficacy data, we adopt a Bayesian data augmentation (BDA) approach (Daniels and Hogan, 2008; Little and Rubin, 2014) to iteratively impute the missing data using the available auxiliary information (See Supplementary Material for detailed sampling steps). We sample from the posterior distribution of the model parameters based on the dataset completed using the imputed values. In the MM study, low-grade toxicity may be observed quickly, and some bioactivity variables are measured repeatedly. We use this auxiliary information to impute missing values of $X_{i}$ and $Y_{i}$, so that adaptive treatment decisions can be made in real time based on the completed dataset. The BDA algorithm iterates between two steps: an imputation (I) step, and a posterior (P) step, in which posterior samples of the parameters are simulated based on the imputed data. Liu et al. (2013) and Jin et al. (2014) used a similar data augmentation approach in dose-finding studies with incompletely observed outcomes. However, they only used follow-up time and did not exploit auxiliary variables to help impute missing outcomes.

We carry out the BDA procedure for posterior sampling using JAGS via the R2jags package (Su and Yajima, 2015). The posterior samples obtained from JAGS can be utilized directly to calculate the posterior utility functions as specified in Section 3. In general, it takes approximately $2 \mathrm{~s}$ in $\mathrm{R}$ to complete one BDA procedure with three chains and a total of 9,000 posterior samples. When the sample size is 180 and the posterior estimates are estimated for each subtype after a cohort of three patients have been treated, it requires about 6 minutes to simulate one trial. We thus used a high performance computing cluster to conduct runs in parallel across 200 computational nodes.

\section{Trial Design}

\subsection{Utility Function}

We take a utility-based approach to quantify efficacy-toxicity risk-benefit trade-offs. To do this, a numerical utility $U(x, y)$ is elicited for each of the elementary outcome 
pairs $(x, y)=(0,0),(1,0),(0,1)$, or $(1,1)$. A consistent utility function must satisfy the admissibility constraints $U(1,0)<\min \{U(1,1), U(0,0)\}$ and $\max \{U(1,1), U(0,0)\}<$ $U(0,1)$. It is convenient to fix the best case utility $U(0,1)=100$ and the worst case utility $U(1,0)=0$, although this is not necessary, and ask the clinicians to specify the utilities $U(0,0)$ and $U(1,1)$ between 0 and 100 for the two remaining intermediate outcome combinations. If $U(1,1)>U(0,0)$, then efficacy is considered more important than toxicity, while if $U(1,1)<U(0,0)$, then avoiding toxicity matters more than achieving efficacy. For illustrations of the choice of $U(x, y)$, see Houede et al. (2010), Thall and Nguyen (2012), or Yuan et al. (2016).

For each disease subtype $b$, the mean utility of regime $r=(d, s)$ given $\boldsymbol{\theta}$ is

$$
\begin{aligned}
u(\boldsymbol{\theta}, b, r) & =\mathrm{E}\{U(X, Y) \mid b=b, r=(d, s), \boldsymbol{\theta}\}= \\
& =\sum_{x=0}^{1} \sum_{y=0}^{1} U(x, y) \operatorname{Pr}\{X=x, Y=y \mid b=b, r=(d, s), \boldsymbol{\theta}\} .
\end{aligned}
$$

The posterior probability that the treatment regime $r=(d, s)$ achieves the highest utility within schedule $s$ is

$$
\omega(b, r)=\operatorname{Pr}\left[u(\boldsymbol{\theta}, b, r)=\max _{r^{\prime} \in\{(1, s), \ldots,(D, s)\}}\left\{u\left(\boldsymbol{\theta}, b, r^{\prime}\right)\right\} \mid \boldsymbol{\Theta}, \tilde{D}_{n}^{\text {obs }}\right],
$$

where $\boldsymbol{\Theta}=\left(\boldsymbol{\theta}_{0}, \boldsymbol{\theta}_{1}, \boldsymbol{\theta}_{2}\right)$. The probability of assignment to regime $r=(d, s)$ under the proposed AR procedure, which is given in detail below, depends on $\omega(b, r)$. This is different from the AR procedures used by Thall and Nguyen (2012), Lee et al. (2015), and others, where the AR probabilities are defined in terms of posterior mean utilities, i.e., $\tilde{\omega}(b, r)=\mathrm{E}\left[u(\boldsymbol{\theta}, b, r) \mid \boldsymbol{\Theta}, \tilde{D}_{n}^{\text {obs }}\right]$. While $\tilde{\omega}(b, r)$ summarizes only the first moment of the posterior of $u(\boldsymbol{\theta}, b, r)$, the probability $\omega(b, r)$ is more variable because it accounts for the posterior distribution of $u(\boldsymbol{\theta}, b, r)$. Hence, AR probabilities defined in terms of $\omega(b, r)$ lead to more extensive exploration of the set of regimes, so $\omega(b, r)$ has a smaller chance of getting stuck at suboptimal regimes.

\subsection{Adaptive Randomization}

Let $\pi_{X}(b, r)=\operatorname{Pr}\{X=1 \mid b, r\}$ be the marginal probability of toxicity and $\pi_{Y}(b, r)=$ $\operatorname{Pr}\{Y=1 \mid b, r\}$ the marginal probability of efficacy, for $b=1, \ldots, B, d=1, \ldots, D$, and $s=1, \ldots, S$, where $r=(d, s)$. For each subtype $b$, we define a set of admissible regimes by adaptively screening out any regimes with either excessively high toxicity or unacceptably low efficacy based on the interim data. For each $b$, denote a fixed elicited upper limit $\bar{\pi}_{X}$ on $\pi_{X}^{T}(b, r)$ and a fixed elicited lower limit $\underline{\pi}_{Y}$ on $\pi_{y}^{E}(b, r)$. The set of admissible regimes $\mathcal{A}_{b}$ for disease subtype $b$ consists of all $r=(d, s)$ that satisfy the two requirements

$$
\operatorname{Pr}\left\{\pi_{X}(b, r)>\bar{\pi}_{X} \mid \Theta, \tilde{D}_{n}^{\text {obs }}\right\}<c_{X} \quad \text { and } \quad \operatorname{Pr}\left\{\pi_{Y}(b, r)<\underline{\pi}_{Y} \mid \Theta, \tilde{D}_{n}^{\text {obs }}\right\}<c_{Y},
$$

where $c_{X}$ and $c_{Y}$ are fixed cutoff probabilities calibrated to obtain a design with good operating characteristics. If no regimes satisfy these admissible criteria, the trial is stopped early and no regime is selected. 
The primary objective of the MM basket trial is to find the optimal admissible treatment regime $r=(d, s)$ for each cancer subtype $b$. Since toxicity assessment is much faster than efficacy assessment, we consider a two-stage trial design. In stage 1, patients in each subtype are randomized fairly among schedules. Since toxicity is observable much earlier than efficacy, the toxicity data play a major role in stage 1 , with treatment regimes that have excessively high toxicity probabilities within a subgroup being ruled out. In stage 2 , as previously missing efficacy outcomes are observed for patients who have completed their efficacy assessment, this efficacy data is included in the decision making. We adaptively randomize the remaining patients among all acceptable regimes across different schedules.

Assume that patients are recruited sequentially to each schedule within each disease subtype. Let $N_{\max }$ be the maximum total sample size, and $p_{b}$ the prevalence of subtype $b=1, \ldots, B$, so $\sum_{b=1}^{B} p_{b}=1$. For each subtype $b$, we fairly allocate $\kappa p_{b} N_{\text {max }}$ patients to each schedule in stage 1 . Thus, the remaining number of patients in stage 2 is (1$\kappa S) p_{b} N_{\max }$. The two-stage trial is conducted as follows.

Stage 1. If the next patient has disease subtype $b$, determine the admissible set $\mathcal{A}_{b}$ based on the most recent data $\tilde{D}_{n}^{\text {obs }}$.

1.1 Randomly choose a schedule, $s$, with probability $1 / S$ each.

1.2 If the selected schedule $s$ has never been tested, then start the subtrial for this schedule at the lowest dose, i.e., $r=(1, s)$. Otherwise, subject to the constraint that no untried dose may be skipped when escalating, randomly choose an admissible regime $r=(d, s) \in \mathcal{A}_{b}, d=1, \ldots, D$, for the next patient with AR probability proportional to $\omega(b, r)$.

1.3 The subtrial for subtype $b$ is either stopped when the maximum sample size $\kappa p_{b} N_{\max }$ is reached, or terminated early if no dose within this schedule is admissible for subtype $b$.

Stage 2. For each newly enrolled patient in subtype $b$, first determine the optimal admissible regime $r_{b}^{*}(s)=\left(d_{b}^{*}(s), s\right)$ that has largest posterior probability of having the maximum mean utility within each $s$, i.e.,

$$
d_{b}^{*}(s)=\underset{d \in\{1, \ldots, D\}}{\arg \max } \omega(b, r) \mathrm{I}\left\{r \in \mathcal{A}_{b} \mid r=(d, s)\right\}, \quad s=1, \ldots, S .
$$

Then choose the schedule $s \in\{1, \cdots, S\}$ with probability proportional to

$$
\omega\left(b, r_{b}^{*}(s)\right)=\operatorname{Pr}\left[u_{\boldsymbol{\theta}}\left(b, r_{b}^{*}(s)\right)=\max _{r_{b}^{\prime} \in\left\{r_{b}^{*}(1), \ldots, r_{b}^{*}(S)\right\}}\left\{u_{\boldsymbol{\theta}}\left(b, r_{b}^{\prime}\right)\right\} \mid \boldsymbol{\Theta}, \tilde{D}_{n}^{\mathrm{obs}}\right],
$$

and assign the new patient to regime $\left(d_{b}^{*}(s), s\right)$. In other words, the second stage adaptively randomizes patients to the best dose-schedule regime within each schedule. Repeat this process until the remaining patients have been treated in the second stage. If no regime is admissible for subtype $b$, then stop the trial for that subtype. 
At the end of the study, based on the complete data $D_{N_{\max }}$, for each subtype $b=1, \ldots, B$, the optimal treatment regime $r_{b}^{*}=\left(d_{b}^{*}, s_{b}^{*}\right)$ is defined as that with the largest posterior probability of having the maximum mean utility among all the regimes, formally

$$
r_{b}^{*}=\underset{r \in \mathcal{A}_{b}}{\arg \max } \operatorname{Pr}\left[u(\boldsymbol{\theta}, b, r)=\max _{r^{\prime}}\left\{u_{\boldsymbol{\theta}}\left(b, r^{\prime}\right)\right\} \mid \boldsymbol{\Theta}, \tilde{D}_{n}^{\mathrm{obs}}\right] .
$$

\subsection{Design Parameter Calibration}

To obtain a design with good performance, one must carefully calibrate the numerical values of both the hyperparameters, $\boldsymbol{\Theta}=\left(\boldsymbol{\theta}_{0}, \boldsymbol{\theta}_{1}, \boldsymbol{\theta}_{2}\right)$, and the design parameters, $\left(N_{\max }\right.$, $\left.\kappa, \bar{\pi}_{X}, \underline{\pi}_{Y}, c_{X}, x_{Y}\right)$. In general, this can be done as follows. The method requires prior values of $\pi_{X}(b, r)$ and $\pi_{Y}(b, r)$ to be elicited from the physicians who are planning the trial, for each subgroup $b$ and regime $r$.

The method uses the idea of prior expected sample size (ESS) given by Morita et al. (2008) to specify the hyperparameters $\boldsymbol{\theta}_{0}, \boldsymbol{\theta}_{1}, \boldsymbol{\theta}_{2}$. As the first step of an iterative process, fix the initial values of $\left(\sigma_{\xi}^{2}, \sigma_{\eta}^{2}, \Sigma_{u, v}\right)$ and $\left(\nu_{\xi}^{2}, \nu_{\eta}^{2}, \psi_{0}, \gamma_{0}\right)$. Given a regime $r$, the values of $\xi_{0}$ and $\eta_{0}$ can be obtained by matching the mode of the prior of each $\pi_{X}(b, r)$ and $\pi_{Y}(b, r)$ with the corresponding elicited value. Using the initial fixed hyperparameters, prior samples of $\pi_{X}(b, r)$ and $\pi_{Y}(b, r)$ values are generated using the hierarchical model (2.1). Following the approach of Lee et al. (2015), each prior sample is fit to a $\operatorname{Beta}(a, b)$ distribution using the method of moments, with the prior ESS approximated as $a+b$. The hyperparameter $\boldsymbol{\theta}_{0}$ can be calibrated repeatedly until $a+b$ is near 1 for each $(b, r)$ combination, which gives a reasonably vague prior. A similar procedure can be used to obtain $\boldsymbol{\theta}_{1}$ and $\boldsymbol{\theta}_{2}$. Additional details, including guidelines for choosing the numerical prior and design parameter values, are given in Section S2 of Supplementary Material.

The upper limit $\bar{\pi}_{X}$ on $\pi_{X}(b, r)$, and the lower limit $\underline{\pi}_{Y}$ on $\pi_{Y}(b, r)$ also must be specified by the clinician. In practice, the maximum sample size $N_{\max }$ of a phase I-II trial is specified based on practical limitations, and choosing $N_{\max }$ may be informed by preliminary trial simulations to assess the design's performance for a range of different feasible values. We recommend that at least $D$ patients are assigned to each schedule in stage 1 for each subtype, to ensure that each dose within each schedule has a reasonable probability of being tried, unless the lowest dose is found to be unsafe for that schedule. Formally, this implies that $\kappa \geq \max _{b \in\{1, \ldots, B\}}\left\{D /\left(p_{b} N^{\max }\right)\right\}$. For example, given $N_{\max }=$ $180, D=3, C=3$, and $\left(p_{1}, p_{2}, p_{3}\right)=(3 / 12,4 / 12,5 / 12), \kappa$ should be greater than $1 / 15$, to ensure that at least one patient in subtype 1 can be assigned to each dose for each schedule in stage 1 . When $N_{\max }$ is adequate, we recommend allocating more patients to stage 1, to ensure that the preliminary estimate of the subgroup-specific optimal treatment regime for each subtype is reasonably accurate. In the MM study, $N_{\max }=180$, and we assign at least $3 D=9$ patients to each schedule for each subtype in stage 1. 


\section{Simulation Study}

\subsection{Simulation Design}

A simulation study to assess the proposed design's performance was designed to be similar to the MM trial, with $B=3$ different subtypes and patients in each subtype assigned to one of the nine dose-schedule regimes, for a total of 27 subtype-specific dose-schedule regimes. The toxicity window was $T_{X}=30$ days with the same upper limit $\bar{\pi}_{X}=.15$ for all $\pi_{X}^{T}(b, r)$. Low-grade toxicity was defined as grade 1 or 2 toxicity observed immediately. Bioactivity was simulated as a longitudinal variable measured on each of days $10,20, \cdots, 90$. Assuming equal subgroup proportions $\left(p_{1}, p_{2}, p_{3}\right)=(1 / 3,1 / 3,1 / 3)$, the maximum sample size $N_{\max }=180$ leads to an average of 6.6 patients assigned to each of the 27 subgroup-regime combinations. We also performed a sensitivity analysis to investigate the design's performance for other $\left(p_{1}, p_{2}, p_{3}\right)$ vectors. We set $\kappa=0.15$ so that the stage 1's sample size was 81 . We assumed an accrual rate of 10 patients per month, with patients arriving according to a Poisson process, so it took approximately 18 months to accrue 180 patients. The proposed design was examined under 12 different scenarios. The assumed fixed marginal probabilities $\left(\pi_{X}(b, r), \pi_{Y}(b, r)\right)$ in each scenario are given in Table S1 of the Supplementary Material, and the true mean utilities $\mathrm{E}(U(X, Y) \mid b, r)$ are given in Table S2 of the Supplementary Material. The 12 scenarios have six homogeneous cases and six corresponding heterogeneous cases. For each homogeneous case, say scenario $i, \pi_{Y}(b, r)$ was the same across subtypes $b=1,2,3$ for all $r$. For the corresponding heterogeneous case, $\left\{\pi_{X}(b, r): b=1,2,3\right\}$ and $\pi_{Y}(1, r)$ were the same as in scenario $i$, but $\pi_{Y}(b, r)$ 's were different for $b=2,3$. Comparing the design's performance under scenarios $i$ and $i+1$ thus shows what is gained by borrowing information across subtypes. Let $u_{b}^{\max }$ denote the largest expected utility for all 9 regimes in subtype $b$. The set of subgroup-specific optimal treatment regimes (OTRs) are defined as those have expected utilities no less than $u_{b}^{\max }-5$, so in particular more than one $r$ may be nominally "optimal." The OTRs for each subtype under each scenario are indicated in boldface in Table S2 of the Supplementary Material. The data-generating algorithms for the toxicity and efficacy outcomes $\left(x_{i}, y_{i}\right), i=1, \ldots, n$, and for the bioactivity and low-grade toxicity data, are provided in the Supplementary Material.

In the simulations, we denote the proposed basket phase I-II trial design as $\mathrm{BTD}_{12}$. The detailed configuration of the $\mathrm{BTD}_{12}$ is provided in Supplementary Material. For comparison, we simulated a simpler naive design that is used quite often in practice. This naive design collapses the different disease subtypes into a single population, based on the assumption of homogeneity. To deal with delayed outcomes, this design would make adaptive treatment assignment decisions for new patients only after the outcomes of the previously treated patients are all observed. To evaluate the advantage of borrowing information across subtypes by the $\mathrm{BTD}_{12}$ design, as a comparator we also simulated a design that conducts separate, independent trials for each of the three subtypes, with maximum sample size $p_{b} N_{\max }$ for each subtype $b=1, \ldots, B$. This design, hereafter referred to as ITD $_{12}$, does not borrow information across subtypes for decision making. To evaluate the gain for borrowing information from additional bioactivity and lowgrade toxicity data, we simulated the observed-data version of the $\mathrm{BTD}_{12}$ (denoted by 
$\mathrm{BTD}_{12}^{\mathrm{O}}$ ). This design does not borrow any information from additional bioactivity or low-grade toxicity data. In addition, it makes treatment decisions based on the patients whose outcomes have been completely observed by the decision-making time. As a benchmark for comparison, we also simulated the complete-data version of the $\mathrm{BTD}_{12}$ (denoted by $\mathrm{BTD}_{12}^{\mathrm{C}}$ ), which repeatedly suspends the accrual of new patients prior to each treatment assignment, to wait until all pending outcomes of previously treated patients have been observed completely. As a result, $\mathrm{BTD}_{12}^{\mathrm{C}}$ has no missing outcomes. However, $\mathrm{BTD}_{12}^{\mathrm{C}}$ requires a very lengthy trial duration and is not feasible in practice. Nonetheless, it provides an upper bound for evaluating the performance of the proposed design since $\mathrm{BTD}_{12}^{\mathrm{C}}$ uses all the data in decision making. Lastly, we include the design proposed in Lin et al. (2020a) for ordered cancer subtypes (hereafter referred to as OTD) in the simulation study. The OTD design requires strong prior information on the efficacy probability ordering among different subtypes. To adapt this design in our simulation setting, we assume that the treatment efficacy probabilities follow the ordering: subtype $3>$ subtype $2>$ subtype 1 . For each design, the posterior distributions were updated after each cohort of three patients was treated. We simulated each design 1000 times under each scenario.

\subsection{Simulation Results}

The simulation results of the proposed $\mathrm{BTD}_{12}$ design are summarized in Tables $\mathrm{S} 3$ and S4 of the Supplementary Material, including within-subtype selection percentages and number of patients allocated to each regime. The simulation results show that $\mathrm{BTD}_{12}$ has particularly high probabilities of selecting the optimal subgroup-specific treatment regimes, and it allocates most patients to the appropriate regimes. As mentioned, the marginal toxicity and efficacy probabilities are the same for subtype 1 in scenarios $i$ and $i+1$, for $i$ an odd number, with the key difference that scenario $i$ is a homogeneous case and scenario $i+1$ is a heterogeneous case. Comparing the OTR selection percentages for subgroup 1 for each scenario pair $i$ and $i+1$ shows that the $\mathrm{BTD}_{12}$ design generally has a greater probability of OTR identification, primarily because it borrows information across subtypes in the homogeneous cases.

We next focus on comparisons among the $\mathrm{BTD}_{12}$, naive, $\mathrm{ITD}_{12}$, OTD, and $\mathrm{BTD}_{12}^{\mathrm{C}}$ designs. Table 1 shows the percentages of selecting OTRs for each design. Table 2 provides other operating characteristics, including the percentage of patients allocated to overly toxic regimes with $\pi_{X}(b, r)>\bar{\pi}_{X}$, the percentage of patients allocated to inefficacious regimes with $\pi_{Y}(b, r)<\underline{\pi}_{Y}$, the percentage of trials selecting overly toxic regimes, summed across subgroups (so the maximum value is $300 \%$ ), the average trial duration, and the trial efficiency index, defined as EI $=\sum_{b=1}^{B} p_{b} \frac{\hat{u}_{b}-\bar{u}_{b}}{u_{b}^{\max }-\bar{u}_{b}}$, where $\hat{u}_{b}$ is the empirical expected utility induced by one design for cancer subtype $b, u_{b}^{\max }$ is the maximum utility among the regimes for subtype $b$, and $\bar{u}_{b}$ is the empirical mean utility induced by uniformly allocating patients to each of the dose-schedule regimes within subtype $b$. EI has a maximum value of 1 , and measures how efficient the design is in treating the patients enrolled in the trial: If a design allocates as many patients as possible to the best treatment regime with $\mathrm{E}(U(X, Y) \mid b, r)=u_{b}^{\max }$, then its EI would approach one. Thus, larger EI corresponds to better design performance. Alternatively, if 


\begin{tabular}{|c|c|c|c|c|c|c|c|c|c|c|c|c|c|}
\hline \multirow{2}{*}{ Method } & \multicolumn{12}{|c|}{ Scenarios } & \multirow{2}{*}{ Avera } \\
\hline & 1 & 2 & 3 & 4 & 5 & 6 & 7 & 8 & 9 & 10 & 11 & 12 & \\
\hline \multicolumn{14}{|c|}{ Cancer subtype 1} \\
\hline $\mathrm{BTD}_{12}$ & 91.7 & 80.1 & 80.4 & 70.2 & 73.4 & 47.8 & 85.7 & 72.3 & 75.9 & 69.1 & 85.1 & 84.8 & 76.4 \\
\hline Naiv & 96.6 & 43.2 & 85.6 & 49.8 & 69.3 & 19.5 & 92.8 & 46.5 & 83.5 & 64.2 & 93.2 & 91.0 & 9.6 \\
\hline $\mathrm{ITD}_{12}$ & 73.4 & 73.2 & 62.5 & 65.0 & 39.2 & 37.5 & 61.1 & 67.5 & 53.4 & 56.8 & 67.8 & 68.6 & 60.5 \\
\hline OTD & 93.6 & 64.0 & 78.5 & 60.7 & 74.5 & 33.6 & 87.0 & 59.5 & 77.4 & 67.2 & 89.2 & 91.3 & 73.0 \\
\hline $\mathrm{BTD}_{12}^{\mathrm{O}}$ & 91.6 & 74.3 & 81.2 & 68.0 & 68.8 & 45.8 & 85.8 & 68.4 & 72.5 & 69.1 & 85.4 & 85.4 & 74.7 \\
\hline $\mathrm{BTD}_{12}^{\mathrm{C}}$ & 92.9 & 79.4 & 81.9 & 73.5 & 70.3 & 46.8 & 86.9 & 64.5 & 78.6 & 69.9 & 87.0 & 87.1 & 76.6 \\
\hline
\end{tabular}

Cancer subtype 2

$\begin{array}{llllllllllllll}\text { BTD }_{12} & 93.6 & 76.8 & 79.4 & 70.5 & 73.9 & 61.3 & 84.9 & 81.6 & 74.2 & 79.3 & 87.6 & 86.6 & 79.1\end{array}$

$\begin{array}{llllllllllllll}\text { Naive } & 96.6 & 18.9 & 85.6 & 18.6 & 69.3 & 26.3 & 92.8 & 47.1 & 83.5 & 48.2 & 93.2 & 91.0 & 64.3\end{array}$

$\begin{array}{llllllllllllll}\text { ITD }_{12} & 76.0 & 77.0 & 62.6 & 70.5 & 37.5 & 58.9 & 61.4 & 93.1 & 56.1 & 67.6 & 67.2 & 74.2 & 66.8\end{array}$

$\begin{array}{llllllllllllll}\text { OTD } & 95.1 & 37.5 & 78.8 & 35.2 & 77.1 & 45.0 & 88.7 & 89.4 & 77.9 & 54.0 & 91.0 & 91.7 & 71.8\end{array}$

$\begin{array}{llllllllllllll}\mathrm{BTD}_{12}^{\mathrm{O}} & 91.3 & 72.8 & 80.2 & 66.1 & 70.6 & 62.2 & 84.0 & 81.0 & 74.8 & 74.4 & 86.2 & 88.1 & 77.7\end{array}$

$\begin{array}{llllllllllllll}\mathrm{BTD}_{12}^{\mathrm{C}} & 93.9 & 73.2 & 81.6 & 73.6 & 70.0 & 59.6 & 85.9 & 91.6 & 80.5 & 79.1 & 87.4 & 87.3 & 80.3\end{array}$

Cancer subtype 3

\begin{tabular}{lccccccccccccc} 
BTD $_{12}$ & 92.8 & 77.0 & 79.6 & 84.4 & 73.6 & 73.2 & 84.0 & 53.2 & 76.3 & 51.2 & 85.3 & 83.9 & 76.2 \\
Naive & 96.6 & 3.6 & 85.6 & 66.4 & 69.3 & 32.5 & 92.8 & 19.3 & 83.5 & 41.0 & 93.2 & 91.0 & 67.4 \\
ITD $_{12}$ & 73.9 & 74.5 & 64.7 & 70.9 & 35.5 & 65.5 & 62.9 & 52.1 & 53.4 & 37.9 & 67.6 & 66.5 & 60.5 \\
OTD & 91.6 & 55.6 & 77.4 & 65.5 & 74.1 & 52.4 & 85.5 & 33.4 & 72.8 & 37.0 & 86.5 & 88.6 & 68.4 \\
BTD $_{12}^{\mathrm{O}}$ & 94.2 & 77.4 & 80.8 & 77.2 & 70.0 & 68.7 & 84.1 & 49.1 & 74.3 & 52.4 & 86.2 & 85.0 & 75.0 \\
BTD $_{12}^{\mathrm{C}}$ & 93.6 & 81.0 & 82.0 & 84.7 & 70.8 & 69.7 & 87.2 & 52.1 & 80.5 & 55.9 & 87.0 & 85.5 & 77.5 \\
\hline
\end{tabular}

Table 1: Percentages of selecting optimal treatment regimes within each cancer subtype, for each of the five designs under each of the 12 scenarios in Table S1 of the Supplementary Material. Efficacy is assessed at day 90, and the assessment period for toxicity is 30 days. The accrual rate is 10 patients per month. Scenarios given in boxes correspond to heterogeneous cases. The subgroup-specific optimal treatment regimes are defined as those have expected utilities no less than $u_{b}^{\max }-5$, where $u_{b}^{\max }$ denotes the largest expected utility for all 9 regimes in subtype $b$.

$\mathrm{EI}<0$, then the design is unacceptable since it performs worse than the equal allocation scheme.

Table 1 shows that, in the homogeneous scenarios indexed by odd numbers, on average the naive design achieves the best performance. This is because the homogeneity assumption of the naive design is correct in these scenarios. The naive design thus can be treated as the oracle design in the homogeneous scenarios. However, in the heteroge- 
neous scenarios where the model is misspecified, the naive design has the smallest OTR selection percentage, on average. Across the 12 scenarios, the within-subtype OTR selection percentage by $\mathrm{BTD}_{12}$ is $76 \%$, on average, which exceeds that provided by $\mathrm{ITD}_{12}$ by approximately $15 \%$. The advantage of $\mathrm{BTD}_{12}$ over $\mathrm{ITD}_{12}$ is quite large in the homogeneous scenarios, because $\mathrm{BTD}_{12}$ borrows information across subtypes while $\mathrm{ITD}_{12}$ does not. For example, in scenarios 1,7 , and $9, \mathrm{BTD}_{12}$ has more than a $20 \%$ greater chance of identifying the OTRs than $\mathrm{ITD}_{12}$. The most striking case is scenario 5 , in which the correct OTR selection percentage of $\mathrm{BTD}_{12}$ is almost double that of $\mathrm{ITD}_{12}$.

In the heterogeneous scenarios, indexed by even numbers, one concern is that excessive borrowing of information between subgroups may harm the performance of $\mathrm{BTD}_{12}$. The simulations show that $\mathrm{BTD}_{12}$ still outperforms $\mathrm{ITD}_{12}$ in most of the heterogeneous scenarios, which may be attributed to the ability of $\mathrm{BTD}_{12}$ to adaptively determine the amount of information borrowed from each subtype. For example, in scenario 4, subtype 2 has two OTRs, regimes $(1,1)$ and $(2,3)$, that are totally different for subtypes 1 and 3. The simulation results from Table 1, and Tables S3 and S4 in the Supplementary Material, together, show that $\mathrm{BTD}_{12}$ is able to correctly detect this heterogeneity and allocate most patients to subtype-specific OTRs. The OTR selection percentage for subtype 2 is 70.5 , which is particularly close to that based on $\operatorname{ITD}_{12}$. In addition, since subtypes 1 and 3 have a common OTR, as a result, $\mathrm{BTD}_{12}$ performs better than $\mathrm{ITD}_{12}$ in terms of the OTR selection percentages for subtypes 1 and 3 , due to adaptive information borrowing. There is only one OTR for each subtype, and the three subtype-specific OTRs are different in scenario 6 , which is difficult for $\mathrm{BTD}_{12}$ as information borrowing across subtypes may lead to incorrect OTR selections. However, the simulations show that, in scenario $6, \mathrm{BTD}_{12}$ still is superior to $\mathrm{ITD}_{12}$. Another interesting result is seen in scenario 12 , where the treatment effects are heterogeneous but the locations of the OTRs are the same across the three subtypes. In this case, it appears that $\mathrm{BTD}_{12}$ benefits greatly from information borrowing, since it yields higher OTR selection percentages than $\mathrm{ITD}_{12}$. Moreover, since the toxicity outcomes are assumed to be homogeneous across subtypes, there are always safety advantages from information sharing by $\mathrm{BTD}_{12}$. Borrowing toxicity information across subtypes improves the reliability of the rules for screening out overly toxic regimes, whereas the $\mathrm{ITD}_{12}$, which does not borrow information, has worse safety. This is shown by Table 2, which indicates that $\mathrm{BTD}_{12}$ selects fewer overly toxic regimes and allocates fewer patients to overly toxic regimes, compared to $\mathrm{ITD}_{12}$. Table 2 also shows that $\mathrm{BTD}_{12}$ uniformly dominates the $\mathrm{ITD}_{12}$ design in terms of trial efficiency and average trial duration.

Comparing the operating characteristics of the $\mathrm{BTD}_{12}$ design and the observed-data $\mathrm{BTD}_{12}^{\mathrm{O}}$ design shows that borrowing information from bioactivity and low-grade toxicity data makes the $\mathrm{BTD}_{12}$ more efficient. Table 2 shows that the $\mathrm{EI}$ values of $\mathrm{BTD}_{12}$ are generally larger than those of $\mathrm{BTD}_{12}^{\mathrm{O}}$, and $\mathrm{BTD}_{12}$ allocates fewer patients to overly toxic treatment regimes. In addition, $\mathrm{BTD}_{12}$ yields higher selection percentages of optimal treatment regimes than $\mathrm{BTD}_{12}^{\mathrm{O}}$, especially in the heterogeneous scenarios $2,4,6$, and 8. Recall that the complete data design $\mathrm{BTD}_{12}^{\mathrm{C}}$ is a benchmark that could never be used in practice since, by repeatedly suspending accrual, it would require an impossibly long trial duration. For example, $\mathrm{BTD}_{12}^{\mathrm{C}}$ would require up to 180 months (15 years) to complete a trial of 180 patients. Since the decisions of $\mathrm{BTD}_{12}$ are made based on 


\begin{tabular}{|c|c|c|c|c|c|c|c|c|c|c|c|c|c|}
\hline \multirow{2}{*}{ Method } & \multicolumn{12}{|c|}{ Scenarios } & \multirow{2}{*}{ Average } \\
\hline & 1 & 2 & 3 & 4 & 5 & 6 & 7 & 8 & 9 & 10 & 11 & 12 & \\
\hline \multicolumn{14}{|c|}{$\%$ selection of overly toxic regimes } \\
\hline $\mathrm{BTD}_{12}$ & 1.4 & 1.4 & 1.1 & 7.3 & 4.3 & 2.6 & 13.3 & 41.1 & 49.7 & 53.5 & 12.2 & 18.0 & 17.2 \\
\hline Naive & 0.0 & 0.9 & 0.0 & 3.3 & 3.3 & 0.3 & 6.0 & 27.9 & 32.4 & 32.4 & 6.0 & 11.7 & 10.4 \\
\hline $\mathrm{ITD}_{12}$ & 14.8 & 15.3 & 10.4 & 19.4 & 16.7 & 11.0 & 40.2 & 45.8 & 115.1 & 110.7 & 33.3 & 36.4 & 39.1 \\
\hline OTD & 0.9 & 3.2 & 1.7 & 8.9 & 4.3 & 2.1 & 12.8 & 39.6 & 52.7 & 55.0 & 10.1 & 13.5 & 17.1 \\
\hline $\mathrm{BTD}_{12}^{\mathrm{O}}$ & 1.0 & 2.8 & 0.6 & 10.0 & 4.0 & 2.4 & 16.8 & 42.8 & 53.6 & 55.2 & 16.1 & 20.4 & 18.8 \\
\hline $\mathrm{BTD}_{12}^{\mathrm{C}}$ & 0.4 & 1.4 & 1.9 & 5.2 & 3.6 & 2.2 & 12.6 & 39.9 & 39.6 & 50.5 & 11.8 & 15.9 & 15.4 \\
\hline \multicolumn{14}{|c|}{ \# patients allocated to overly toxic regimes } \\
\hline $\mathrm{BTD}_{12}$ & 16.2 & 16.0 & 15.7 & 22.0 & 9.5 & 6.5 & 22.1 & 30.2 & 81.2 & 79.7 & 17.5 & 18.9 & 28.0 \\
\hline Naive & 12.2 & 12.4 & 12.2 & 18.2 & 6.8 & 4.1 & 17.8 & 25.4 & 74.0 & 74.9 & 12.4 & 14.0 & 23.8 \\
\hline $\mathrm{ITD}_{12}$ & 27.7 & 27.4 & 28.6 & 32.2 & 7.6 & 6.5 & 18.2 & 22.0 & 93.1 & 93.4 & 16.0 & 16.9 & 32.6 \\
\hline OTD & 12.3 & 12.2 & 12.7 & 16.2 & 5.9 & 4.7 & 14.7 & 17.2 & 51.6 & 51.1 & 10.9 & 11.3 & 18.4 \\
\hline $\mathrm{BTD}_{12}^{\mathrm{O}}$ & 20.4 & 19.4 & 19.6 & 26.9 & 10.1 & 7.6 & 24.5 & 31.4 & 84.6 & 84.5 & 19.8 & 19.8 & 30.7 \\
\hline $\mathrm{BTD}_{12}^{\mathrm{C}}$ & 14.8 & 14.2 & 13.3 & 19.8 & 8.3 & 5.6 & 20.2 & 27.5 & 74.5 & 76.0 & 15.1 & 15.1 & 25.4 \\
\hline \multicolumn{14}{|c|}{ \# patients allocated to subtherapeutic regimes } \\
\hline $\mathrm{BTD}_{12}$ & 9.9 & 12.1 & 37.8 & 43.6 & 9.2 & 10.6 & 47.5 & 53.5 & 21.4 & 27.7 & 64.6 & 70.2 & 34.0 \\
\hline Naive & 9.0 & 17.1 & 32.9 & 59.2 & 7.9 & 9.0 & 41.2 & 72.0 & 19.8 & 33.1 & 58.3 & 62.8 & 35.3 \\
\hline $\operatorname{ITD}_{12}$ & 22.0 & 19.1 & 61.0 & 48.8 & 21.8 & 21.4 & 72.4 & 66.1 & 26.6 & 27.2 & 83.2 & 94.9 & 45.2 \\
\hline OTD & 5.2 & 8.1 & 21.7 & 27.7 & 4.5 & 5.2 & 28.7 & 35.8 & 9.2 & 16.1 & 38.3 & 42.7 & 20.3 \\
\hline $\mathrm{BTD}_{12}^{\mathrm{O}}$ & 10.9 & 12.1 & 39.4 & 42.2 & 9.7 & 10.3 & 50.4 & 54.6 & 19.3 & 26.2 & 64.3 & 71.8 & 34.3 \\
\hline $\mathrm{BTD}_{12}^{\mathrm{C}}$ & 9.0 & 11.3 & 33.8 & 43.0 & 7.7 & 9.4 & 45.2 & 53.5 & 20.7 & 26.8 & 62.5 & 66.6 & 32.4 \\
\hline \multicolumn{14}{|c|}{ Trial efficiency index } \\
\hline $\mathrm{BTD}_{12}$ & 53.0 & 46.5 & 53.2 & 43.2 & 27.0 & 26.7 & 35.5 & 19.1 & 36.4 & 37.1 & 37.3 & 36.2 & 37.6 \\
\hline Naive & 56.9 & 38.2 & 57.9 & 36.8 & 27.5 & 19.6 & 42.5 & 5.1 & 40.9 & 36.2 & 45.3 & 42.3 & 37.4 \\
\hline $\operatorname{ITD}_{12}$ & 28.0 & 27.0 & 35.4 & 33.6 & 26.8 & 14.9 & 19.6 & 17.1 & 24.6 & 27.2 & 25.8 & 23.2 & 25.3 \\
\hline OTD & 41.9 & 33.2 & 44.6 & 29.2 & 18.6 & 16.8 & 28.7 & 6.4 & 29.5 & 27.3 & 38.3 & 30.6 & 21.0 \\
\hline $\mathrm{BTD}_{12}^{\mathrm{O}}$ & 46.0 & 40.4 & 49.0 & 38.2 & 21.3 & 23.1 & 31.3 & 17.2 & 33.6 & 32.8 & 35.4 & 34.8 & 33.6 \\
\hline $\mathrm{BTD}_{12}^{\mathrm{C}}$ & 54.0 & 48.6 & 56.6 & 45.7 & 26.8 & 26.9 & 38.7 & 22.1 & 40.0 & 39.9 & 40.5 & 39.8 & 40.0 \\
\hline \multicolumn{14}{|c|}{ Average trial duration (in months) } \\
\hline $\mathrm{BTD}_{12}$ & 21.0 & 21.0 & 21.0 & 21.0 & 21.0 & 21.0 & 21.0 & 21.0 & 21.0 & 21.0 & 21.0 & 21.0 & 21.0 \\
\hline Naive & 180.0 & 180.0 & 180.0 & 180.0 & 180.0 & 180.0 & 180.0 & 180.0 & 180.0 & 180.0 & 180.0 & 180.0 & 180.0 \\
\hline $\operatorname{ITD}_{12}$ & 27.0 & 27.0 & 27.0 & 27.0 & 27.0 & 27.0 & 27.0 & 27.0 & 27.0 & 27.0 & 27.0 & 27.0 & 27.0 \\
\hline OTD & 21.0 & 21.0 & 21.0 & 21.0 & 21.0 & 21.0 & 21.0 & 21.0 & 21.0 & 21.0 & 21.0 & 21.0 & 21.0 \\
\hline $\mathrm{BTD}_{12}^{\mathrm{O}}$ & 24.0 & 24.0 & 24.0 & 24.0 & 24.0 & 24.0 & 24.0 & 24.0 & 24.0 & 24.0 & 24.0 & 24.0 & 24.0 \\
\hline $\mathrm{BTD}_{12}^{\mathrm{C}}$ & 180.0 & 180.0 & 180.0 & 180.0 & 180.0 & 180.0 & 180.0 & 180.0 & 180.0 & 180.0 & 180.0 & 180.0 & 180.0 \\
\hline
\end{tabular}

Table 2: Operating characteristics based on five designs under the 12 scenarios in Table S1 of the Supplementary Material, assuming accrual rate of 10 patients per month. Scenarios given in boxes correspond to heterogeneous cases. "\% selection of overly toxic regimes" is the sum of the percentages of selecting overly toxic regimes for all three subtypes, so the maximum value is $300 \%$. 
less data, unavoidably, it is less efficient than the optimal complete-data $\mathrm{BTD}_{12}^{\mathrm{C}}$ design, according to the EI values in Table 2. In terms of other metrics, such as OTR selection or overdose control, $\mathrm{BTD}_{12}$ and $\mathrm{BTD}_{12}^{\mathrm{C}}$ have very similar performance, however. Although outcomes are missing early in the trial, it appears that $\mathrm{BTD}_{12}$ is able to recover from the efficiency loss in the late-stage of the trial when more outcomes that had been temporarily missing are observed.

Comparing the $\mathrm{BTD}_{12}$ basket design and the order-based OTD design, we found that these two methods yield similar OTR selection percentages in homogenous scenarios (indexed by odd numbers), where the ordering restriction of OTD was not violated. However, in heterogenous scenarios (indexed by even numbers) where the true subtype-efficacy structure does not satisfy the strong ordering restriction on the treatment efficacy probabilities among the three cancer subtypes, the performance of OTD is uniformly inferior to that of $\mathrm{BTD}_{12}$. An interesting finding is that OTD generally yields smaller numbers of patients at overly toxic or subtherapeutic regimes than $\mathrm{BTD}_{12}$, as noted in Table 2. This is potentially due to the fact that OTD puts highly informative priors on the efficacy probabilities, causing extensive information borrowing across different cancer subtypes. As a consequence, the convergence of parameter estimates based on OTD is faster, and thus OTD can quickly identify overly toxic or subtherapeutic regimes. But the accompanying risk with such a faster convergence rate is the higher chance of being trapped in suboptimal treatment regimes. On the other hand, BTD $_{12}$ does not assume a strong association among cancer subtypes, and uses observed data to adaptively determine the level of information sharing. At the beginning of the trial when the information contained in the observed data is sparse, $\mathrm{BTD}_{12}$ tends to be more exploratory and test more untried regimes. Therefore, it has a higher number of patients treated at overly toxic of subtherapeutic regimes than $\mathrm{BTD}_{12}$. Nevertheless, $\mathrm{BTD}_{12}$ is much safer than the independent $\mathrm{ITD}_{12}$ design. Furthermore, $\mathrm{BTD}_{12}$ uses more information, leading to a higher EI than OTD across all scenarios. This in turn implies that more patients are treated at optimal or nearly optimal regimens based on $\mathrm{BTD}_{12}$.

\subsection{Sensitivity Analyses}

We carried out sensitivity analyses to assess the robustness of the $\mathrm{BTD}_{12}$ design, by considering different (a) prevalence proportions for the three subgroups, (b) sample sizes for stage 1 while keeping $N_{\max }=180$ constant, (c) patient accrual rates, and (d) prior distributions on the heterogeneity parameters. In each sensitivity analysis, the other simulation configurations were unchanged from those in Section 4.1. In this section, we only describe the results (see Figure 1) under scenarios 1-4 of Table S1 in the Supplementary Material, since the substantive conclusions based on the other scenarios are the same.

In sensitivity assessment (a), we considered three prevalence ratios. The first two were $p_{1}: p_{2}: p_{3}=3: 4: 5$, which enrolls more patients with subtype 3 , and $p_{1}: p_{2}:$ $p_{3}=5: 4: 3$, which enrolls more patients with subtype 1 . Additionally, since in Table 5 of Fonseca et al. (2009) MM patients are classified as \{hyperdiploid, non-hyperdiploid, other\} with respective percentages $45,40,15$, we examined the design's behavior using 
the corresponding values $p_{1}: p_{2}: p_{3}=9: 8: 3$. The simulation results show that, when the treatment effects are homogeneous (scenarios 1 and 3), the OTR selection percentages for the proposed design are not sensitive to the different prevalence ratios. However, when there are heterogeneous treatment effects, as in scenarios 2 and 4, the subtype-specific OTR selection percentage increases with the sample size of the subtype. For the most extreme imbalance $p_{1}: p_{2}: p_{3}=9: 8: 3$, lower OTR selection percentages of about $60 \%$ are seen in Scenario 2 and $70 \%$ in Scenario 4, which are slightly below the values for the case $p_{1}: p_{2}: p_{3}=5: 4: 3$, although the decrements are very small in Scenarios 1 and 3 .

In sensitivity assessment (b), we evaluated the design under three different stage 1 sample sizes, $N_{1}=54,81$, and 108 , corresponding to $\kappa=0.10,0.15$, or 0.20 , since $N_{1}=\kappa N_{\max } B$. The simulations suggest that the OTR selection percentage is not sensitive to these rather large differences in stage 1 sample size. However, we also found that smaller $\kappa$ results in a larger EI (results not shown). This is because, when $\kappa$ is small, more patients are enrolled in stage 2, which is the optimization stage. As a result, a design with a smaller value of $\kappa$ generally allocates more patients to OTRs, and hence is more efficient in this regard.

In sensitivity assessment (c), we examined accrual rates of 6,10 , and 15 patients per month, which lead to respective average trial durations of 33, 21, and 15 months. Given the fixed toxicity/efficacy assessment windows, the accrual rate determines the amount of missing data at the time of decision making. The faster new patients arrive, the more likely it will be that patients treated previously will have missing outcomes that must be imputed. The simulation results displayed in panel (c) of Figure 1 show that the OTR selection percentage for the proposed method is quite robust to this range of accrual rates. However, the faster the accrual rate, the larger the amount of missing data in the decision-making process. Although the accrual rate does not affect the OTR selection percentage for $\mathrm{BTD}_{12}$, additional simulations (results not provided) show that a fast accrual rate would make the proposed method less efficient and more aggressive.

In sensitivity assessment (d), we evaluated the effects of different prior distributions on the heterogeneity parameters $\tau_{\eta}$ and $\tau_{w}$, which play critical roles in determining the amount of information borrowing between subtypes. We considered three cases: $\tau_{\eta}, \tau_{w} \stackrel{\text { i.i.d. }}{\sim}$ half-Cauchy $(0,1), \tau_{\eta}, \tau_{w} \stackrel{\text { i.i.d. }}{\sim}$ half-Cauchy $(0,5)$, and $\tau_{\eta}, \tau_{w} \stackrel{\text { i.i.d. }}{\sim} \operatorname{IG}(0.1,0.1)$. The half-Cauchy $(0,1)$ prior places more probability mass on the homogeneous case, i.e., $\tau_{\eta}=\tau_{w}=0$. The simulation results based on these three prior specifications are particularly close, suggesting that our design is not sensitive to these prior distributions.

\section{Concluding Remarks}

The proposed phase I-II basket trial design finds the optimal subtype-specific doseschedule by first assuming a three-level hierarchical model. Complications due to lateonset toxicity or efficacy outcomes are addressed by using a two-stage design with adaptive randomization, which is a natural approach to this problem. In stage 1, when most of the efficacy data are unavailable, toxicity data can be utilized for decision making to screen out unsafe treatment regimes. When more patients have completed their 
(a) Sensitivity to different prevalence ratios

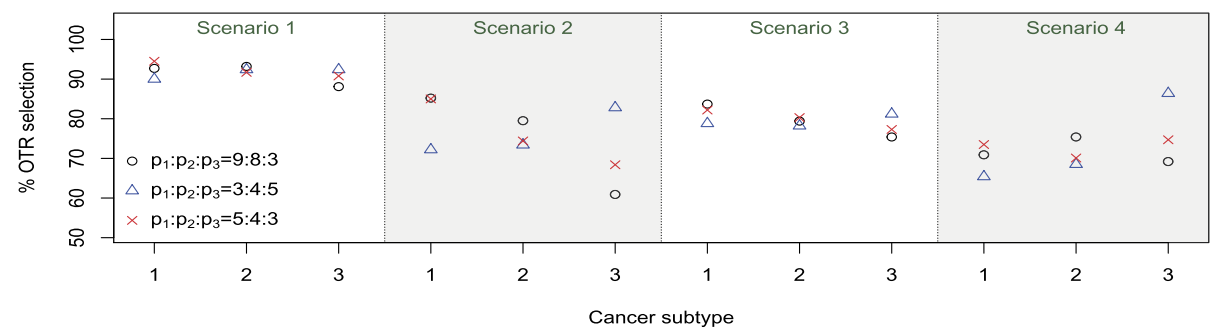

(b) Sensitivity to different stage 1 sample sizes, $\mathrm{N}_{1}$, in a $180-$ patient trial

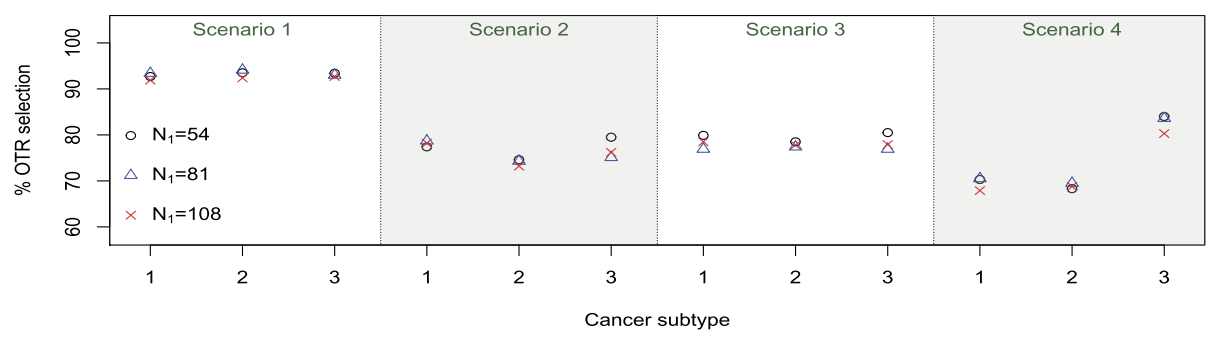

(c) Sensitivity to different patient accrual rates

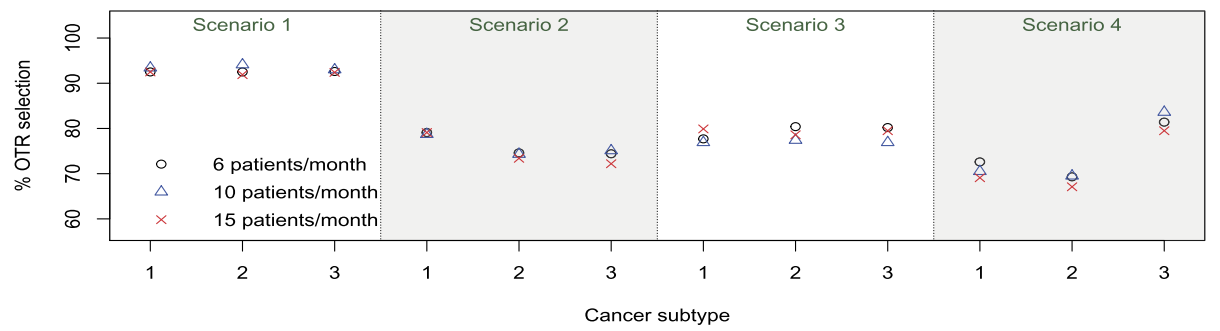

(d) Sensitivity to different prior distributions on $\tau_{\eta}$ and $\tau_{w}$

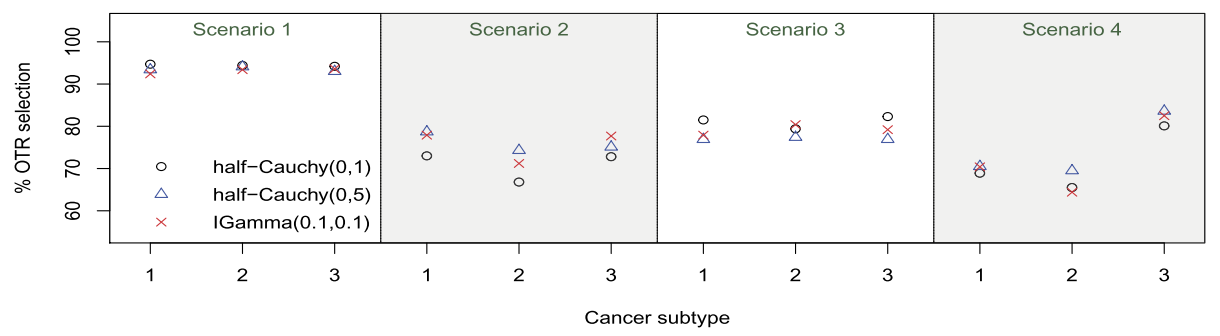

Figure 1: Sensitivity assessments of the proposed $\mathrm{BTD}_{12}$ method to (a) different prevalence ratios $\left(p_{1}: p_{2}: p_{3}\right)$; (b) different stage 1 sample sizes; (c) different patient accrual rates; (d) different prior distributions on $\tau_{\eta}$ and $\tau_{w}$. The sensitivity assessments are conducted based on scenarios 1-4 of Table S1 in the Supplementary Material. 
follow-up in stage 2, the efficacy outcome plays a major role in treating the remaining patients, and for choosing optimal (dose, schedule) regimes. To deal with different cancer subtypes, the Bayesian hierarchical model assumes that the dose-schedule treatment effects for different subtypes vary around a common mean, and thus facilitates adaptive shrinkage based on the observed data. The simulations show that the proposed design uniformly outperforms an approach that conducts separate independent trials within subgroups when the regime effects are homogeneous across subtypes. In addition, the operating characteristics of the proposed design are very close to those of the benchmark complete-data design, indicating that the efficiency loss due to missing data is minimal.

Although the assumed imputation models for missing values of $X_{i}$ and $Y_{i}$ may be incorrect, this will have negligible effects on the design's performance, for several reasons. First, the imputation model only provides partial/indirect information, and the treatment-assignment decisions of the proposed method are mainly determined by the inference model. Second, outcomes are only temporarily missing. Once patients with pending outcomes have finished their entire assessments, temporarily unobserved outcomes become available and contribute to the estimation of the primary inference model (2.1). Third, the primary objective of the trial is not to obtain accurate inference on the subtype-specific regime-response relationships, but rather to identify optimal subtype-specific treatment regimes. Our simulations show that, even with misspecified imputation models, the proposed design still does a good job of allocating patients to optimal regimes and provides high probabilities of making correct selections.

While the Bayesian hierarchical model adaptively borrows information across cancer subtypes, a caveat is that it tends to shrink the subtype-specific treatment effects toward the common mean, which may lead to incorrect treatment assignment decisions when there is a mixture of homogeneous and heterogeneous subgroups. As suggested by an associate editor, we have considered four scenarios where the treatment effects are very similar for some subtypes and very different for the other subtypes. We compared the operating characteristics of $\mathrm{BTD}_{12}$ with those of the naive design, which is based on the subtype homogeneity assumption, and those of the $\mathrm{ITD}_{12}$ design, which is based on the subtype heterogeneity assumption. The simulation results given in the Supplementary Material show that the proposed $\mathrm{BTD}_{12}$ design strikes a balance between the fullinformation-borrowing naive design and the no-information-borrowing ITD $_{12}$ design. However, in this case, one may hypothesize that the performance of $\mathrm{BTD}_{12}$ might be improved by adaptively combining or splitting the cancer subtypes using the latent subgroup membership variable approach of Chapple and Thall (2018). This is a potential area for future research.

\section{Supplementary Material}

Supplementary material of "A Phase I-II Basket Trial Design to Optimize Dose-Schedule Regimes Based on Delayed Outcomes" (DOI: 10.1214/20-BA1205SUPP; .pdf). The Supplementary Material contains Bayesian data augmentation steps, the prior elicitation procedure, simulation configurations, and additional simulation results. 


\section{References}

Albert, J. H. and Chib, S. (1993). Bayesian analysis of binary and polychotomous response data. Journal of the American Statistical Association 88, 669-679. MR1224394. doi: https://doi.org/10.1080/01621459.1993.10476321. 183

Berry, S. M., Broglio, K. R., Groshen, S., and Berry, D. A. (2013). Bayesian hierarchical modeling of patient subpopulations: efficient designs of phase II oncology clinical trials. Clinical Trials 10, 720-734. doi: https://doi .org/10.1177/1740774513497539. 180

Chapple, A. G. and Thall, P. F. (2018). Subgroup-specific dose finding in phase I clinical trials based on time to toxicity allowing adaptive subgroup combination. Pharmaceutical Statistics 17, 734-749. doi: https://doi.org/10.1002/pst.1891. 181, 199

Chen, M. H. and Dey, D. K. (1998). Bayesian modeling of correlated binary responses via scale mixture of multivariate normal link functions. Sankhya: The Indian Journal of Statistics, Series A 60, 322-343. MR1718864. 183

Cheung, Y. K. and Chappell, R. (2000). Sequential designs for phase I clinical trials with late-onset toxicities. Biometrics 56, 1177-1182. MR1815616. doi: https://doi . org/10.1111/j.0006-341X.2000.01177.x. 181

Chib, S. and Greenberg, E. (1998). Analysis of multivariate probit models. Biometrika 85, 347-361. doi: https://doi.org/10.1093/biomet/85.2.347. 183

Chu, Y., and Yuan, Y. (2018a). A Bayesian basket trial design using a calibrated Bayesian hierarchical model. Clinical Trials 15, 149-158. doi: https://doi.org/ 10.1177/1740774518755122. 180

Chu, Y. and Yuan, Y. (2018b). BLAST: Bayesian latent subtype design for basket trials accounting for patient heterogeneity. Journal of the Royal Statistical Society: Series $C$ (Applied Statistics). MR3787974. doi: https://doi.org/10.1111/rssc.12255. 180

Cunanan, K. M., Iasonos, A., Shen, R., Begg, C. B., and Gönen, M. (2017). An efficient basket trial design. Statistics in Medicine 36, 1568-1579. MR3631980. doi: https : // doi.org/10.1002/sim.7227. 180

Daniels, M. J. and Hogan, J. W. (2008). Missing Data in Longitudinal Studies: Strategies for Bayesian Modeling and Sensitivity Analysis. CRC Press. MR2459796. doi: https://doi.org/10.1201/9781420011180. 187

Durie, B. G., Harousseau, L. J., Miguel, J. S., et al. (2006) International uniform response criteria for multiple myeloma Leukemia 20, 1467-1473. doi: https://doi . org/10.1038/sj.leu.2404284. 186

Fonseca, R., Bergsagel, P. L., Drach, J., et al. (2009) International myeloma working group molecular classification of multiple myeloma: spotlight review. Leukemia 23, 2210-2221. doi: https://doi.org/10.1038/leu.2009.174. 180, 196

Gelman, A. (2006). Prior distributions for variance parameters in hierarchical models. Bayesian Analysis 1, 515-534. MR2221284. doi: https://doi.org/10.1214/ 06-BA117A. 184 
Houede, N., Thall, P. F., Nguyen, H., Paoletti, X., and Kramar, A. (2010). Utility-based optimization of combination therapy using ordinal toxicity and efficacy in phase III trials. Biometrics 66, 532-540. MR2758833. doi: https://doi.org/10.1111/j. $1541-0420.2009 .01302 . x . \quad 188$

Jin, I. H., Liu, S., Thall, P. F., and Yuan, Y. (2014). Using data augmentation to facilitate conduct of phase I-II clinical trials with delayed outcomes. Journal of the American Statistical Association 109, 525-536. MR3223730. doi: https://doi.org/ 10.1080/01621459.2014.881740. 182, 187

Lee, J., Thall, P. F., Ji, Y., and Müller, P. (2015). Bayesian dose-finding in two treatment cycles based on the joint utility of efficacy and toxicity. Journal of the American Statistical Association 110, 711-722. MR3367259. doi: https://doi.org/10.1080/ 01621459.2014.926815. 188,190

Lee, J., Thall, P.F., and Rezvani, K. (2018) Optimizing natural killer cell doses for heterogeneous cancer patients based on multiple event times. Journal of the Royal Statistical Society, Series C, 68, 461-474. MR3903004. doi: https://doi.org/10. 1111/rssc.12271. 181

Lin, R., Thall, P. F., and Yuan, Y. (2020a). An adaptive trial design to optimize doseschedule regimes with delayed outcomes. Biometrics, 76, 304-315. doi: https://doi . org/10.1111/biom.13116. 181, 192

Lin, R., Thall, P. F., and Yuan, Y. (2020b). A Phase I-II Basket Trial Design to Optimize Dose-Schedule Regimes Based on Delayed Outcomes - Supplementary Material. Bayesian Analysis. doi: https://doi.org/10.1214/20-BA1205SUPP. 182

Little, R. J. and Rubin, D. B. (2014). Statistical Analysis With Missing Data. John Wiley \& Sons. MR1925014. doi: https://doi.org/10.1002/9781119013563. 187

Liu, S., Yin, G., and Yuan, Y. (2013). Bayesian data augmentation dose finding with continual reassessment method and delayed toxicity. The Annals of Applied Statistics 7, 2138-2156. MR3161716. doi: https://doi.org/10.1214/13-A0AS661. 185, 187

Morita, S., Thall, P. F., and Müller, P. (2008). Determining the effective sample size of a parametric prior. Biometrics 64, 595-602. MR2432433. doi: https://doi.org/ 10.1111/j.1541-0420.2007.00888.x. 190

Morita, S., Thall, P. F., and Takeda, K. (2017). A simulation study of methods for selecting subtype-specific doses in phase 1 trials. Pharmaceutical Statistics 16, 143156. doi: https://doi.org/10.1002/pst.1797. 181

Ornes, S. (2016). Core Concept: Basket trial approach capitalizes on the molecular mechanisms of tumors. Proceedings of the National Academy of Sciences 113, 70077008. doi: https://doi.org/10.1073/pnas.1608277113. 180

Redig, A. J. and Jänne, P. A. (2015). Basket trials and the evolution of clinical trial design in an era of genomic medicine. Journal of Clinical Oncology 33, 975-977. doi: https://doi.org/10.1200/JC0.2014.59.8433. 180

Simon, R. and Roychowdhury, S. (2013). Implementing personalized cancer genomics in 
clinical trials. Nature Reviews Drug Discovery 12, 358-369. doi: https://doi.org/ 10.1038/nrd3979. 179

Simon ,R., Geyer, S., Subramanian, J., and Roychowdhury, S. (2016). The Bayesian basket design for genomic variant driven phase II trials. Seminars in Oncology 43,16. doi: https://doi.org/10.1053/j.seminoncol.2016.01.004. 180

Su, Y. S., and Yajima, M.(2015). Package 'R2jags'. R package version 0.5-7, https:// cran.r-project.org/web/packages/R2jags/index.html 187

Thall, P. F., Wathen, J. K., Bekele, B. N., Champlin, R. E., Baker, L. H., and Benjamin, R. S. (2003). Hierarchical Bayesian approaches to phase II trials in diseases with multiple subtypes. Statistics in Medicine 22, 763-780. doi: https://doi.org/10. 1002/sim.1399. 180

Thall, P. F. and Nguyen, H. Q. (2012). Adaptive randomization to improve utility-based dose-finding with bivariate ordinal outcomes. Journal of Biopharmaceutical Statistics 22, 785-801. MR2931071. doi: https://doi.org/10.1080/10543406.2012.676586. 188

Thall, P. F., Nguyen, H. Q., Braun, T. M., and Qazilbash, M. H. (2013). Using joint utilities of the times to response and toxicity to adaptively optimize scheduledose regimes. Biometrics 69,673-682. MR3106595. doi: https://doi.org/10.1111/ biom.12065. 180

Thall, P. F., Nguyen, H. Q., and Zinner, R. G. (2017). Parametric dose standardization for optimizing two-agent combinations in a phase I-II trial with ordinal outcomes. Journal of the Royal Statistical Society: Series C (Applied Statistics) 66, 201-224. MR3611684. doi: https://doi.org/10.1111/rssc.12162. 182

Trippa, L. and Alexander, B. M. (2017). Bayesian baskets: a novel design for biomarkerbased clinical trials. Journal of Clinical Oncology 35, 681-687. doi: https://doi. org/10.1200/JC0.2017.35.4_suppl.681. 180

Yuan, Y., Nguyen, H. Q. and Thall, P. F. (2016). Bayesian Designs for Phase I-II Clinical Trials. Chapman \& Hall/CRC: New York. 180, 188

Yuan, Y. and Yin, G. (2011). Bayesian phase I-II adaptively randomized oncology trials with combined drugs. Annals of Applied Statistics 5, 924-942. MR2840181. doi: https://doi.org/10.1214/10-AOAS433. 181

Zhang, W., Sargent, D. J., and Mandrekar, S. (2006). An adaptive dose-finding design incorporating both toxicity and efficacy. Statistics in Medicine 25, 2365-2383. MR2240943. doi: https://doi.org/10.1002/sim.2325. 180

\section{Acknowledgments}

We thank the handling Editor, the Associate Editor, the two referees, and the Editor for their many constructive and insightful comments that have led to significant improvements in the article. 